\title{
Fundación FIEL frente a las políticas de Estado en Educación Superior en Argentina $(1989-2001)^{*}$
}

\author{
FIEL Foundation facing State policies in Argentina's Higher \\ Education (1989-2001)
}
Fundação FIEL frente às políticas de Estado para a Educação Superior na Argentina (1989-2001)

Enzo Andrés Scargiali**

\begin{abstract}
RESUMEN
El artículo tiene por objeto caracterizar y problematizar las propuestas de reforma del sistema de educación superior argentino realizadas por la Fundación de Investigaciones Económicas Latinoamericanas (FIEL) durante el proceso de reforma del Estado, que tuvo lugar durante el gobierno de Carlos Menem (1989-1999) en Argentina. En primer lugar, se caracteriza el ciclo neoliberal argentino y el rol de los think tanks en el contexto del diseño de políticas públicas durante esta etapa. En segundo término, se reconstruyen las trayectorias de los expertos de Fundación FIEL. Luego, se describen las investigaciones y actividades que realizaron en torno a las políticas en educación superior. Por último, se busca aproximar una descripción de la forma en que se produce la circulación de ideas generadas por este think tank para el ámbito estatal y la forma en que estos discursos interpelaron a los actores de las universidades nacionales durante la década en indagación.
\end{abstract} Palabras clave: Fundación FIEL, neoliberalismo, políticas en educación superior, think tanks.

\footnotetext{
* La investigación fue financiada a través del proyecto UBACyT “Think tanks, agenda de "cambio" y política en Argentina, Paraguay y Colombia (2003-2019)" dirigido por la Dra. Lorena Soler. Programación científica 2018-2019 de la Universidad de Buenos Aires, Argentina.

** Argentino. Licenciado en Sociología, Universidad de Buenos Aires, Argentina. Consejo Nacional de Investigaciones Científicas y Técnicas (CONICET) e Instituto de Estudios de América Latina y el Caribe, Universidad de Buenos Aires (IEALC-UBA). Buenos Aires, Argentina. escargiali@sociales.uba.ar
} 


\begin{abstract}
This article seeks to describe and problematize reform proposals of the Argentinean higher education system made by the Latin American Economic Research Foundation (FIEL) during Carlos Menem's government's State reform process (1989-1999) in Argentina. First, we characterize the Argentine neoliberal cycle and the role of think tanks in the context of public policy design during this stage. Secondly, we reconstruct the trajectories of FIEL Foundation experts. Then, we describe the research and activities carried out around higher education policies. Finally, we describe the circulation of ideas generated by this think tank for the state sphere and how these discourses questioned the actors of the national universities during the decade under investigation.
\end{abstract}

\section{RESUMO}

O objetivo deste artigo é caracterizar e problematizar as propostas de reforma do sistema de educação superior argentino feitas pela Fundação de Pesquisas Econômicas Latino-americanas (FIEL) durante o processo de reforma de Estado ocorrido no governo de Carlos Menem (1989-1999) na Argentina. Primeiramente, caracteriza-se o ciclo neoliberal argentino e o papel dos think tanks no contexto do desenvolvimento de políticas públicas durante esta etapa. Em segundo lugar, são reconstruídas as trajetórias dos especialistas da Fundação FIEL. Em seguida, são descritas as pesquisas e atividades realizadas em torno das políticas de educação superior. Por último, procura-se aproximar uma descrição da forma em que se produz a circulação de ideias geradas por este think tank para o setor estatal e a maneira na qual estes discursos interpelaram os atores das universidades nacionais durante a década em investigação.
Keywords: FIEL Foundation, neoliberalism, higher education policies, think tanks.

Palavras-chave: Fundação FIEL, neoliberalismo, políticas de educação superior, think tanks. 


\section{Introducción}

El artículo tiene por objeto caracterizar y problematizar las propuestas de reforma del sistema de educación superior argentino realizadas por la Fundación de Investigaciones Económicas Latinoamericanas (FIEL) durante el proceso de reforma del Estado que tuvo lugar durante el gobierno de Carlos Menem (1989-1999) en Argentina.

Durante la década de 1990 cobra fuerza, por un lado, la democracia liberal en un sentido instrumental y, por otro, la totalización del mercado (Ansaldi y Soler, 2015) que posibilita al régimen neoliberal la presencia de un Estado activo y beligerante que responda a aquel (Escalante, 2016).

Las discusiones acerca del rol de Estado en la sociedad y su relación con el mercado se remontan al siglo XVIII. Sin embargo, el interés de los economistas por la educación en general y el sistema universitario, en particular, tiene un origen reciente que se ancla hacia la década de 1960:

Si bien la preocupación por el crecimiento económico y las causas que hacen que unos países sean más ricos que otros data del propio origen de la teoría económica, el descubrimiento y estudio sistemático de que la educación juega un rol importante en el desarrollo se inició hace cuarenta años. (Tenti Fanfani, 2004, p. 17).

Desde mediados de la década de 1970, con el advenimiento del neoliberalismo, caracterizado por un proceso de concentración y centralización del capital, las estructuras de la política y el Estado se vieron copadas por "profesionales" y "expertos" en el diseño de políticas públicas, entre las que se encontraban las discusiones en torno al sistema educativo. En en este marco, cobraron relevancia las recomendaciones de los expertos en economía, provenientes de diferentes organismos multilaterales y think tanks (en adelante TT) que comenzaron a expandirse en América Latina. Como destacan Brunner y Sunkel (1995), este proceso se da en el marco de una mayor presión hacia la racionalización y tecnificación de la toma de decisiones a nivel estatal y la adopción y ejecución de políticas.

Entendiendo que los TT son uno de los engranajes clave para la circulación de ideas y en particular durante el neoliberalismo, para el diseño de políticas públicas, el trabajo se propone: en primer lugar, ca- 
racterizar el ciclo neoliberal argentino y el rol de los TT en el contexto del diseño de políticas públicas durante esta etapa. En segundo lugar, se reconstruyen las trayectorias de los expertos de Fundación FIEL. Luego, se describen las investigaciones y actividades que realizaron en torno a las políticas en educación superior. Por último, se busca aproximar una descripción de la forma en que se produce la circulación de ideas generadas por el TT para el ámbito estatal y la forma en que estos discursos interpelaron a los actores de las universidades nacionales durante la década en indagación.

\section{Metodología}

El artículo indaga en una primera etapa la consolidación de las derechas latinoamericanas en el gobierno, y se centra en el caso argentino, en el momento de imposición de políticas de Estado de corte neoliberal caracterizado por los siguientes rasgos: la afirmación de la democracia liberal en un sentido instrumentalista y la defensa del mercado como vastamente han estudiado Soler et al. (2019), Ansaldi (2017), Ansaldi y Soler (2015) y Giordano (2014) 1 .

En función de los objetivos propuestos, se realiza un análisis desde la perspectiva de la sociología histórica - que permite la hibridación de dos disciplinas: la sociología y la historia-. Es propia de esta hibridación una concepción del tiempo como categoría histórica y como perspectiva metodológica (Ansaldi y Giordano, 2012). En este sentido, permite analizar los hechos únicos a partir de la historia dentro de modelos propios de la sociología (Bonnell, 1980). A los efectos de este artículo, dicha perspectiva permite articular la reconstrucción histórica con el análisis de los actores sociales, políticos y económicos. Además, con el foco puesto en las reformas del sistema de educación superior, se hace hincapié en los estudios en sociología de la educación en Argentina en el contexto neoliberal (Filmus, 1996; Tenti Fanfani, 2004; Unzué y Emiliozzi, 2013).

1 El artículo forma parte de los resultados alcanzados por el Proyecto "Think tanks, agenda de "cambio" y política en Argentina, Paraguay y Colombia (2003-2019)" dirigido por la Dra. Lorena Soler con sede en la Universidad de Buenos Aires entre los años 2018 y 2019. Durante estos años, el equipo de investigación se centró en realizar un análisis comparativo de las redes de expertos que aglutinan los TT y cómo sus ideas impactan en la construcción de una agenda pública de "cambio" en Argentina, Colombia y Paraguay. 
El recorte de análisis temporal se centra en los años 1989 a 2001, en pos de estudiar la formulación de recomendaciones publicados por Fundación FIEL en el contexto del predominio de gobiernos del periodo neoliberal en Argentina, en particular, las presidencias de Carlos Menem (1989-1995 y 1995-1999). Como se analiza en el siguiente apartado, durante estos años se produjeron profundas reformas dentro del sistema de educación superior del país. Por otro lado, se reconstruyeron las trayectorias de economistas de FIEL a partir de la interacción de acciones significativas en su contexto o condiciones de producción, con el objeto de dar cuenta de las dimensiones estructurales del problema y los aspectos coyunturales (Braudel y Mendoza, 1970)².

Por último, el artículo se nutre de la revisión de textos bibliográficos especializados en la relación entre TT y política en América latina y el análisis documental de informes de Fundación FIEL: La descentralización de la educación superior: elementos de un programa de reforma (1993), Una educación para el siglo XXI (2000), Educación universitaria. Aportes para el debate acerca de su efectividad y equidad (2003) y otras publicaciones del Estado Nacional: Decreto 506/93, Decreto 2427/93, Informes de Gestión de Ministerio de Cultura y Educación de la Nación (Del Bello, 1996a; 1996b). Para la sistematización y el análisis de datos se apeló a las técnicas de fichado, resumen y construcción de tipologías que permiten ordenar los núcleos temáticos de los casos relevados y su análisis y crítica (Carrera, 1976).

\section{Desarrollo}

La expansión del neoliberalismo en Argentina durante el gobierno de Carlos Menem (1989-1999)

Durante las décadas de 1980 y 1990 América Latina atravesó profundos cambios institucionales y económicos. Como ha destacado Dreyfus (2012) la crisis de las finanzas públicas que afectó a las economías

2 Un primer análisis se puede encontrar en Scargiali (2018) donde se caracterizó a los expertos de Fundación FIEL y se realizó un recorrido por las principales temáticas trabajadas y las recomendaciones hechas al Estado para su reforma. En el presente artículo, se presentan resultados ligados con las recomendaciones en políticas de educación superior y la forma en que son analizados los datos producidos por el propio Estado en cuanto al sistema de educación superior. 
nacionales durante estos años condujo a los gobiernos a realizar sus gastos de otra forma, partiendo de la premisa de que los recursos disponibles deben ser empleados en función de un criterio de productividad, o mejor aún, que las decisiones públicas deben ser tomadas sobre la base del análisis costo-beneficio.

En este marco, el 8 de julio de 1989 Carlos Menem asumió la presidencia de la Nación Argentina, que heredaba la crisis del Estado y una democracia débil tras la última dictadura institucional de las Fuerzas Armadas (1976-1983), pero también la emergencia económica producida por la tendencia hacia la globalización que disminuyó la capacidad de decisión del Estado Nacional (Filmus, 2016). Además, la puesta en cuestión de la centralidad democrática se traducía en la pérdida de confianza hacia su capacidad de asegurar el cumplimiento de las funciones centrales de la sociedad (Portantiero, 1988). En efecto, las limitaciones a la intervención del Estado excedían la situación interna del país, sino que también se encontraba condicionada por factores de decisión externos, donde los organismos internacionales como el Fondo Monetario Internacional (FMI) o el Banco Mundial (BM) cumplían un papel principal.

El gobierno menemista comenzó a implementar una serie de reformas económicas y de desregulación y redimensionamiento del Estado que rápidamente fueron apoyadas por diferentes sectores del empresariado (grandes exportadores, el sector financiero local y acreedores externos). Menem ejecutó las reformas económicas que eran exigidas por los organismos financieros internacionales a cambio de oxígeno político y crediticio para su gobierno: “Cuanto mayor era la presión internacional (...) también mayor resultaba la respuesta simbólica y práctica de adhesión de Menem al liberalismo económico y el recurso a decisiones de autoridad para ejecutar las reformas" (Castiglioni, 1996, p. 13).

Entre los objetivos del plan económico se destacaba, por un lado, el establecimiento de las bases de un régimen monetario y cambiario y, por otro, la construcción de los fundamentos económicos de una coalición social de orden político y electoral que amalgamara los intereses de diferentes sectores sociales (Pucciarelli, 2011).

Si bien el proceso de reforma del Estado comenzó durante el primer gobierno de Carlos Menem, entre 1989-1995, las reformas en las polí- 
ticas de educación superior se produjeron entre 1995 y 1999: periodo conocido como de "modernización burocrática" (Albornoz y Gordon, 2011). Durante este segundo periodo, se introdujeron reformas institucionales en los organismos de ciencia y técnica, y en la relación con la docencia e investigación en las universidades nacionales, con el apoyo de organismos internacionales de crédito como el BID y BM. En julio de 1996, con la asunción de Juan Carlos Del Bello ${ }^{3}$ como secretario de Ciencia y Tecnología e interventor de Conicet, se profundizó el programa de reformas. Del Bello afirmaba en su discurso de asunción de las tareas de la SECyT: "Ciencia y tecnología no se incorporó a proceso de reforma del Estado y de reforma estructural de la sociedad. Se ha avanzado en la democracia, la privatización y la estabilidad, pero esta es una cuenta pendiente" (Ciencia: hora de cuentas, 1996).

\section{Las políticas del Estado Nacional para las universidades nacio- nales}

La década de 1990 marcó la redefinición de la relación entre Estado y universidades nacionales a partir de la agenda marcada por los organismos internacionales de financiamiento (BM y BID). Sus efectos se visualizan en la Ley de Educación Superior (LES) y el Programa de Reforma de la Educación Superior (PRES) sancionadas en 1995 (Gordon, 2018). Sin embargo, la aplicación de políticas en las universidades nacionales no fue una tarea simple por su tradición y uso de la autonomía, lo que las ha convertido en sujetos activos que han logrado sostenerse frente a los embates del Estado neoliberal (Unzué y Emiliozzi, 2013).

En 1993, a través del Decreto 506/93, fue creada la Secretaría de Políticas Universitarias (SPU) en el ámbito del Ministerio de Educación y Justicia de la Nación. Según Albornoz y Gordon (2011), su establecimiento estuvo pensado como un primer paso para la reformulación del vínculo entre las universidades nacionales y el gobierno a partir de

3 Del Bello, un histórico militante político del Partido Justicialista dentro del ámbito universitario, cuya carrera académica siempre se mantuvo ligada a la planificación de educación superior. Durante el gobierno de Carlos Menem, antes de acceder a la cartera de Ciencia y Tecnología y la intervención del Conicet en 1996, había formado parte de la Secretaría de Programación Económica del Ministerio de Economía durante la gestión de Domingo Cavallo y fue autor de la Ley de Educación Superior (LES) sancionada por el Poder Legislativo en 1995. 
una mayor capacidad de intervención. Al mismo tiempo, durante ese año, el Decreto 2427/93, impulsó el programa de incentivos a docentes/investigadores de las universidades nacionales, como un primer intento por generar asignaciones monetarias a partir de mecanismos competitivos y de acreditación de actividades. También, durante 1993 fue creada la Comisión de Acreditación de Posgrado (CAP), la habilitación de un intento de "mercado académico" que, con el financiamiento del BM, a partir de un crédito de más de 165 millones de dólares, se consolidó con la aplicación del Programa de Reforma de Educación Superior (PRES) y la creación, en 1995, de la Comisión Nacional de Evaluación y Acreditación Universitaria (CONEAU) (Albornoz y Gordon, 2011).

Una de las principales transformaciones del ámbito universitario se dio con la sanción de la Ley de Educación Superior (LES) de 1995, a partir de lo cual tuvo lugar la expansión de los posgrados arancelados y la evaluación de las actividades desarrolladas en las universidades. En este sentido, la búsqueda de recursos propios supuso la irrupción de la lógica mercantil y su contracara, las formas precarizadas de contratación de docentes (Unzué, 2013). Como destaca el mismo autor: "la neoliberalización de la universidad argentina fue consecuencia de una clara política que puso en prácticas opciones 'voluntarias' para acceder a recursos escasos, lo que despertó un sorprendente espíritu 'innovador' en el sistema” (Unzué, 2013, p. 28).

\section{Think tanks y expertos durante el neoliberalismo}

El corpus acerca de las investigaciones en torno a la relación entre TT y política en general, y su presencia en América Latina durante el neoliberalismo en particular es amplio. Diferentes investigaciones dan cuenta de sus características e imbricaciones con otros actores políticos y económicos. En este breve apartado, se intenta dar cuenta de algunas de estas discusiones que dan forma al campo de estudios.

Una primera aproximación a la conceptualización de TT permite dar cuenta de su rol como catalizadores entre la academia y la política: "Son instituciones mediadoras entre el mundo de la reflexión (la ciencia) y el mundo de la acción (la política)" (Morales, 2021). El autor identifica cinco roles que cumple este tipo de instituciones: la participación en el debate público; legitimidad y credibilidad basada 
en evidencia científica; aportes a la estabilidad democrática; cuidado de su autonomía e independencia frente a sus vías de financiamiento; y la capacidad de presentar espacios de socialización y acuerdos sociales (Morales, 2021). Sin embargo el autor, haciendo referencia a la investigación Global Think tanks. Policy Networks and Governance (McGann y Whelan, 2020), también advierte acerca de las limitaciones a la autonomía e independencia de los TT frente a los actores políticos y económicos, cuestión que durante la década de 1990 cobra fuerza en las investigaciones acerca de los TT en América Latina ${ }^{4}$.

Uña y Garcé (2006) describen el accionar de los TT latinoamericanos en tres momentos. El primero remite a la década de 1960, cuando se produce la tecnificación del rol de los expertos en economía en América Latina y se difunde la presencia de organismos internacionales. Un segundo periodo, marcado por la última dictadura institucional de las Fuerzas Armadas (1976-1983), donde se fortaleció la figura de los expertos en economía como los portadores de los conocimientos necesarios para comprender y resolver los diversos problemas que atravesaba el Estado. Y el tercer momento que tiene lugar con la apertura del proceso de redemocratización hacia mediados de la década de 1980. Allí, los TT argentinos fueron clave a la hora de proporcionar "el capital social de legitimidad y una justificación académica/intelectual para los planes político-económicos promovidos por el nuevo gobierno democrático" (Mitchell, 2021, p. 63).

En este último momento florecieron las consultoras y espacios mediáticos dedicados al análisis de la coyuntura económica, así como el reclutamiento de equipos técnicos en la función pública (Heredia, 2011) quienes, de la mano de la doctrina "managerialista" desarrollaron sus recomendaciones sobre la base de un postulado doble: por un lado, el precepto de que nada diferencia a los organismos públicos de las empresas privadas; y por otro, el método científico de gestión puede ser aplicado de forma general (Dreyfus, 2012).

4 En relación con este punto abonan los trabajos de investigación realizados por Hernán Ramírez (2007b) acerca de los institutos económicos y acción política en Brasil y Argentina (IPES, FIEL y Fundación Mediterránea) recogidos en "Corporaciones en el poder" y la tesis doctoral "Navegar el Atlántico a contramarea: la Fundación Internacional para la Libertad y la agenda liberal en la ofensiva en América Latina (2002-2016)" (Giménez, 2021). 
Al analizar las particularidades de los TT latinoamericanos, Uña et al. (2004) destacan como una característica central la de ser "grupos de intelectuales motivados políticamente que influyen sobre las burocracias y los tomadores de decisiones en base a su experiencia" (p. 7). Esta formulación dista de cómo se presentan ante el ámbito político y la sociedad en general. Como destaca Camou (1997), los TT son espacios especializados en la creación, distribución y aplicación del saber de forma estratégica en la orientación de políticas en las sociedades latinoamericanas. Estos espacios de construcción y difusión de conocimientos, durante la década de 1990, definieron las estrategias y posibilidades de las instituciones que marcaban la agenda pública (Lorenc Valcarce, 2002). Thompson (1994) los describe en los siguientes seis puntos:

Utilizan metodologías científicas para validar sus saberes, pero no se limitan a temas científicos; son multidisciplinarios; los lazos que construyen van más allá del ámbito científico y se encuentra más cercano al político; definen con un amplio grado de libertad los problemas que deben ser considerados socialmente relevantes y la elaboración de sus recomendaciones; se preocupan por una variedad de problemas de amplio interés; y por último, marcan la agenda de investigación al resto de la comunidad. (p. 10).

En este punto, se acuerda con Mercado (2017) acerca del rol político que cumplen los TT:

Los TT muestran una nueva forma de intervenir en política que, diferenciándose de la tradicional competencia partidaria, incursiona en las organizaciones de redes de experticia tanto a nivel local como internacional (...) Si bien se los presenta como agentes neutrales y ajenos al campo de la política, partimos del supuesto que su producción de conocimiento promueve una conceptualización deshistorizante y desideologizante sobre la democracia y la participación política. (p. 50).

Bonnet (2008) considera que la ideología neoconservadora que acompañó a la hegemonía menemista constituyó una adaptación de las doctrinas forjadas en los TT globales. En este marco, la relación entre los especialistas y la política se incrementó en el marco de las "redes de asuntos", conectando agencias de gobierno, TT, centros de investi- 
gación, fundaciones privadas, universidades, empresas y organismos multilaterales (Camou, 1997). Los TT modificaron el proceso de toma de decisiones en la política, desempeñando un papel clave para los consensos sociales acerca del valor de la democracia, la globalización y la apertura económica externa (Mitchell, 2021).

Con el final de la dictadura institucional de las Fuerzas Armadas (1976-1983), los TT argentinos comenzaron a asesorar técnicamente a gobiernos y partidos ${ }^{5} \mathrm{y}$ han impulsado las reformas neoliberales a partir de la producción y difusión de ideas, datos y argumentos sobre las decisiones que debe tomar el Estado (Botto, 2011). En este sentido, son hacedores de política por fuera de los partidos políticos a partir de las acciones de lobby y consultoría (Fischer y Plehwe, 2013). Los TT se pusieron a la cabeza de una serie de políticas de modernización que los gobiernos debían poner en práctica a partir del modelo de mercado, primero defendido por los economistas de la Escuela de Chicago y luego las instituciones financieras internacionales como el FMI, el BM y la Organización para la Cooperación y el Desarrollo Económico, OCDE (Dreyfus, 2012).

Por otro lado, las privatizaciones de la década de 1990 en Argentina también hicieron pie en la producción de conocimientos que, desplazada de la universidad pública, se trasladó a las fundaciones y TT, vaciando la investigación de sus conocimientos sustantivos y dando mayor relevancia a la producción de conocimiento práctico tendiente a influir en las decisiones. Como ha destacado Mercado (2017), los TT presentan sus resultados como verdades científicas, sin embargo, su producción de conocimiento tiene un sesgo antiestatal y promercado.

En este marco, cumplen un rol fundamental los tecnócratas, o bien, expertos. Bobbio et al. (1994), valiéndose de la ambigüedad conceptual del término, los definen como actores, profesionales que, además de su poseer la capacidad de influir a partir de la consulta técnica, for-

$5 \quad$ Mitchell (2021) indaga en la participación de la Fundación Ford en el financiamiento de proyectos académicos en el Centro de Estudio de Estado y Sociedad, CEDES, y el Centro de Investigaciones Sociales sobre el Estado y la Administración, CISEA, tras el proceso de redemocratización argentino. Los proyectos se encontraban enfocados en: relaciones y economía internacionales, el sector agropecuario de la economía argentina, planificación urbana y regional y el rol de los partidos políticos en el sistema democrático. Con el triunfo de Raúl Alfonsín en 1983, parte de los expertos de CEDES y CISEA pasaron a ocupar cargos importantes en el gobierno nacional. 
man parte de las decisiones de los órganos políticos asumiendo una configuración despolitizada. En otras palabras, los "expertos" toman el lugar de los hombres políticos, quienes ceden terreno ante las decisiones entendidas como el resultado de una serie de cálculos y de presiones científicas (p. 1551).

Neoliberalismo y expertos se implican de forma mutua. Según Morresi (2010), existen elementos teóricos dentro los planteos neoliberales acerca de la forma de difundir e implantar reformas que requieren la acción de "expertos" más que de políticos. En efecto, estos expertos fueron fundamentales para la cimentación de consensos sociales a través de la elaboración de interpretaciones y la construcción de redes de poder (Heredia, 2011). Svampa (2008), amplía la categoría de "expertos" al sugerir que la relación va más allá de su paso por el ámbito político. Por sus espacios de formación y circulación tienen la capacidad de afianzar las relaciones entre el ámbito empresarial, el Estado y los organismos multilaterales.

Los expertos se presentan por fuera de la política, pero su producción de conocimientos no se encuentra disociada del ámbito político que da forma a un nuevo "consenso ideológico" acerca de la mejor forma de aplicar las medidas económicas que llevaría adelante el gobierno menemista.

En efecto, a la vez que los expertos niegan el ámbito político - apelando al fin de las ideologías y de los conflictos de interés de clases- y se enfocan en necesidades puntuales a ser resueltas por técnicos y políticos (Ansaldi y Soler, 2015), elaboran sus ideas, conceptos y propuestas de forma nominal y con facilidad para ser interpretados, lo que los vuelve figuras de confianza (Acosta et al., 2016).

Los expertos, encolumnados dentro de los ámbitos privados de producción de conocimiento como los TT, avanzaron en una nueva serie de marcos para la producción científica de la economía. Como han considerado Brunner y Sunkel (1995), el tipo de investigaciones que llevan adelante difiere de las emprendidas en el ámbito universitario: en general, son coyunturales, de corto alcance y de diagnóstico, con el objeto de brindar legitimidad al discurso neoliberal. Además, estos expertos suelen formar parte de los cuadros técnicos de los Ministerios y organismos (Vommaro, 2014). En Argentina, durante la década 
de 1990, el Estado desarrolló una red de consultores y asesores financiados por agencias internacionales y fundaciones locales que eran contratados por organismos y Ministerios del Estado Nacional para el diseño y ejecución de políticas públicas (Oszlak y Gantman, 1994).

Beltrán (2011), uno de los primeros investigadores en analizar las implicancias de FIEL en el diseño y ejecución de políticas públicas del Estado argentino, ha destacado que, durante la década de 1990, TT y asociaciones del empresariado funcionaron como los principales sostenes del Estado a la hora de diseñar y aplicar políticas tendientes a su reforma y un nuevo tipo de regulación del mercado.

En este marco, durante el periodo bajo análisis, desde FIEL se analizó una serie de documentos de trabajo y publicaciones que exploraban diferentes temáticas relativas a la política económica y social que debía llevar adelante el Estado argentino ante la reforma neoliberal. En particular, este artículo se centra en aquellas recomendaciones que se versaban acerca de la reforma del sistema de educación superior.

\section{Caracterización histórica de Fundación FIEL}

FIEL fue fundada en el año 1964 en la Ciudad de Buenos Aires por las principales corporaciones empresariales del país: la Cámara Argentina de Comercio (CAC), la Sociedad Rural Argentina (SRA), la Unión Industrial Argentina (UIA) y la Bolsa de Comercio de Buenos Aires (Scargiali, 2018). Si bien, el apoyo de estas corporaciones privadas y empresas fue crucial para el surgimiento y consolidación de la institución, su creación se anclaba en las ideas neoliberales de diversos círculos intelectuales de la década de 1960 (Morresi, 2010). Uno de los primeros en esbozar estas ideas fue Alsogaray, quien consideraba que los economistas debían "abandonar su torre de marfil" y "descender a la arena política" (Morresi, 2010, p. 12). Como refuerza Giménez (2021), en la primera etapa de fundación de TT en América Latina durante la década 1960, la organización de FIEL estuvo en consonancia con la difusión de la retórica acerca del libre mercado y el discurso anticomunista.

En línea con ello, Ramírez (2007a) sostiene que la FIEL había sido creada con el objeto de contrarrestar el peso de las políticas desarrollistas o de tendencias populistas que se oponían a los intereses de los grandes grupos económicos. Desde su creación, y principalmente durante 
la década de 1990, muchos de sus cuadros técnicos y expertos dieron su salto a la gestión pública. Principalmente, poblaron el Ministerio de Economía y Finanzas en todas las dictaduras institucionales de las Fuerzas Armadas posteriores a 1966, con excepción del gobierno de facto encabezado por Roberto Levingston durante el breve periodo 1970-1971. Además, han ocupado cargos en la presidencia y vicepresidencia del Banco Central de la República Argentina (BCRA) (Ramírez, 2007a).

FIEL, que se define como una "institución independiente, apolítica y sin fines de lucro" (FIEL, s.f) durante la década de 1990, según consta en la portada de sus publicaciones (FIEL, 1993; 2000), recibió aportes para su funcionamiento de más de 170 empresas locales, multinacionales, privadas y del sector estatal. Además, recibieron financiamiento a partir de actividades de consultoría, estudios e investigaciones contratadas por organismos nacionales, provinciales y locales; la venta de servicios regulares y sus publicaciones (indicadores de coyuntura e industriales y trabajos especiales respecto de la situación económica).

FIEL distribuye su estructura en dos grandes áreas. Por un lado, una dirección ejecutiva conformada por dirigentes empresarios que al mismo tiempo formaban parte también de otras redes de interacción corporativa, como la Cámara de Comercio de los Estados Unidos de Norteamérica (AmCham), la Asociación Cristiana de Dirigentes de Empresas (ACDE) y el Instituto para el Desarrollo Empresarial de la Argentina (IDEA) ${ }^{6}$. Por otra parte, durante el periodo analizado la dirección académica se encontraba a cargo de los economistas Daniel Artana $^{7}$, Juan Luis Bour ${ }^{8}$ y Fernando Navajas ${ }^{9}$. El plantel de expertos se

6 A partir de la revisión de la trayectoria de los miembros de la Dirección ejecutiva de FIEL, cuyo listado era publicado en la primera sección de los documentos de trabajo analizados, pueden verificarse las diferentes adscripciones institucionales.

$7 \quad$ Es Licenciado en Economía por la Universidad Nacional de La Plata y doctor en Economía por la Universidad de California. Entre sus principales tareas se destacan los análisis de coyuntura económica de la Argentina y de América Latina; el estudio de la política fiscal, específicamente la reforma de las pensiones y los estudios sobre regulación de los servicios públicos y defensa de la competencia.

8 Es licenciado en Economía por la Universidad de Buenos Aires y no cuenta con estudios de posgrado. Es consultor de diferentes organismos internacionales y ha publicado diferentes artículos avalados por FIEL y el BID.

9 Es Licenciado en Economía por la Universidad Nacional de La Plata y Doctor en Economía por la Universidad de Oxford. Fue jefe de Gabinete del Ministerio de Economía durante la década de 1990 y economista senior de la Comisión Económica para América Latina y el Caribe (CEPAL) de las Naciones Unidas. 
completaba con 15 economistas, un asistente de investigación y siete técnicos estadísticos, a quienes se sumaban entre cuatro y seis investigadores invitados de forma anual.

Cada uno de los documentos de trabajo publicados era firmado por un equipo que reportaba al Directorio de FIEL. Durante la década de 1990, produjeron 71 documentos que exploran diferentes aristas de las cuestiones económicas y coyunturales más relevantes para la reforma e innovación de la administración del Estado. En particular, sobre la política en educación superior, desarrollaron tres grandes trabajos: $L a$ descentralización de la educación superior: elementos para un programa de reforma (1993); Una educación para el siglo XXI (2000); y Educación universitaria. Aportes para el debate acerca de su efectividad y equidad (2003).

\section{Los expertos de FIEL en educación superior}

En el apartado se avanza en la caracterización de los expertos de la fundación en el ámbito de la educación superior, lo que permite destacar:

En primer lugar, la relevancia de la educación pública en la formación de los expertos. Más de la mitad de los economistas (56\%) que escribieron durante estos años se formaron en la Facultad de Ciencias Económicas de la Universidad de Buenos Aires, seguidos por más de un 30\% que hizo lo propio en la Universidad Nacional de La Plata. Solo en muy pocos casos la formación de grado fue realizada en universidades privadas como Universidad Católica Argentina (5\%), y Universidad Argentina de la Empresa (3\%) (Tabla 1). Además, en el 31\% de los casos, estas carreras fueron el último escalón alcanzado en la formación académica de los expertos de la fundación. Un tercio de los expertos de Fundación FIEL no realizaron cursos de posgrado al momento de redactar los diferentes documentos de trabajo y recomendaciones al Estado en educación superior.

Como afirman Brunner y Sunkel (1995), hacia finales de la década de 1980 la universidad había perdido su aura de reputación tradicional y los expertos se enfrentaban al mercado de conocimientos valiéndose de su posición en el campo y de las redes de las que forman parte en nuevos tipos de instituciones y de su participación en la esfera política. 
Tabla 1

Carreras de formación de grado de los economistas de Fundación FIEL (19892001)

\begin{tabular}{llc}
\hline Institución & Carrera & $\%$ \\
\hline Universidad de Buenos Aires & Licenciatura en Economía & $56 \%$ \\
\hline Universidad Nacional de La Plata & Licenciatura en Economía & $33 \%$ \\
\hline Universidad Católica Argentina & Licenciatura en Economía & $4 \%$ \\
\hline Universidad Argentina de la & Licenciatura en Economía & $3 \%$ \\
\hline Empresa & Ingeniería Comercial & $1 \%$ \\
\hline Universidad de Chile & Licenciatura en Ciencia & \\
\hline Universidad Católica Argentina & Política & $1 \%$ \\
\hline Universidad Nacional de Cuyo & Licenciatura en Sociología & $1 \%$ \\
\hline Total & & $100 \%$ \\
\hline
\end{tabular}

Fuente: Elaboración propia sobre la base de documentos de trabajo de FIEL (1989-2003).

En segundo lugar, se indagó en la relación entre los expertos con diversos organismos internacionales. Analizando la trayectoria de cada uno de ellos, se pudo verificar que casi el $40 \%$ había realizado trabajos por fuera de la organización, contratados por organismos internacionales (Tabla 2). Como han considerado Grassetti y Prego (2017), los TT deben ser analizados a partir de una mirada integral: "abordarlos en el marco de estrategias que superan las fronteras nacionales y hasta regionales. Los vínculos que tienen, las relaciones y acuerdos que construyen dan cuenta de eso" (p. 123).

Tabla 2

Participación de economistas de FIEL en trabajos de consultoría en organismos internacionales

\begin{tabular}{lc}
\hline $\begin{array}{l}\text { Economistas de FIEL con participación en organismos } \\
\text { internacionales }\end{array}$ & $38 \%$ \\
\hline $\begin{array}{l}\text { Economistas de FIEL sin participación en organismos } \\
\text { internacionales }\end{array}$ & $62 \%$ \\
\hline
\end{tabular}

Fuente: Elaboración propia sobre la base de documentos de trabajo de FIEL (1989-2003).

Entre los organismos internacionales que contrataron los servicios de estos especialistas se destacan los dependientes del sistema de la Organización de Naciones Unidas: el Programa de Naciones Unidas para el Desarrollo (PNUD) y la Comisión Económica para América La- 
tina y el Caribe (CEPAL). Además, han asesorado trabajos y han coordinado proyectos para el BID y el BM. Como han considerado Brunner y Sunkel (1995), los investigadores sociales que se desempeñan en organismos internacionales se erigieron en la década de 1990 como personal privilegiado del campo al poder interactuar entre el ámbito privado de producción de conocimientos el ámbito empresarial, el ámbito público (el Estado) y el sector externo (los organismos internacionales).

Los TT generan una comunidad de ideas que ponen en circulación a la hora de definir las estrategias y posibilidades de los agentes e instituciones que marcan la agenda pública. En efecto, la red de expertos de FIEL, durante la década de 1990 funcionó como uno de los principales engranajes para la circulación de ideas económicas, y hasta en algunos casos, parte de ellos ha saltado a la función pública en el ámbito del Ministerio de Economía y también han sido de importancia para el diseño de políticas públicas, en este caso, las referentes a la educación superior.

\section{Diagnóstico y recomendaciones en políticas en educación su- perior de FIEL}

Durante la década de 1990 prevaleció una mirada centrada en la necesidad de eliminar las restricciones que tendía la burocracia estatal sobre el mercado y la sociedad civil (Filmus, 2016). Partiendo de esta premisa, el sistema educativo también era considerado un espacio que debía formar parte del mercado y ser incorporado a las reformas estatales (Del Bello, 1996a). A nivel internacional, este tipo de recomendaciones fueron promovidas por el BM, y a nivel local, se hallan en los trabajos de investigación producidos por Fundación FIEL. En particular, los Documentos de Trabajo $\mathrm{N}^{\circ} 38$ (1993) y $\mathrm{N}^{\circ} 79$ (2003) permiten identificar un hilo que conduce desde las recomendaciones en política de educación superior, a sus alcances a lo largo de la década neoliberal y la realidad que proyectaban los datos del Estado hacia principios de la década de 2000.

Entre las principales consideraciones del Documento de Trabajo $\mathrm{N}^{\circ} 38$ (FIEL, 1993) se proponía desregular "los mercados de los factores productivos de la educación superior en convergencia con las políticas económicas recomendadas por diferentes agencias del ajuste de la oferta y demanda educativa vía el sistema de precios" (FIEL, 1993, 
p. 1). Esta concepción economicista de la educación se sostenía en la idea de que el Estado debía reducir la asignación de recursos de origen fiscal a las universidades nacionales y que debía ser rediscutido y reasignado a partir de los requerimientos de los mercados laborales y del rumbo económico del país.

Por otro lado, los y las estudiantes también eran entendidos como "individuos que tienen la capacidad de adquirir habilidades y conocimientos" (FIEL, 1993, p. 5) que podían ser considerados bienes privados incorporados e internalizados. En línea con ello, el BM en su informe sobre educación superior de 1995 consideraba que "las universidades preparan las capacidades técnicas de alto nivel que constituyen la base del crecimiento económico" (Banco Mundial, 1995). Además, desde FIEL se hacía hincapié en que el eje central de la educación superior y su presencia en la sociedad debía asociarse al entrenamiento para la vida laboral: "El objetivo central de una educación para el mercado pasa a ser el de un centro de formación de profesionales y técnicos superiores de acuerdo con los roles demandados por los puestos de trabajo" (FIEL, 1993, p. 2). Históricamente, el sistema de educación superior argentino se ha sustentado en tres grandes pilares: la formación de profesionales, la investigación científica y las actividades de extensión con la comunidad, que en el plan de reformas de FIEL quedan por fuera de las actividades esenciales que deben ser desarrolladas por las universidades.

El Documento de Trabajo de 1993 dedica una sección al análisis de la participación de la educación privada en el sistema educativo universitario a partir de datos suministrados por el Ministerio de Educación y Cultura de la Nación. En sus líneas, los expertos arriban a la conclusión de que la gratuidad en la educación universitaria pública y las restricciones a la apertura de nuevas instituciones significan una desventaja propiciada por el Estado para la recreación de un mercado educativo competitivo cuya principal consecuencia es "la coacción sobre las posibilidades de elección del alumno" (FIEL, 1993, p.5). En efecto, se reclama por una mayor flexibilidad en las regulaciones para la expansión de la educación privada.

A partir de este diagnóstico, se precisaban algunas "opciones y recomendaciones". Los expertos de FIEL argumentaban que la aplicación de diversos grados de arancelamiento y el financiamiento condiciona- 
do por la demanda permitiría desarrollar una política universitaria que contemplara "la libertad de elección de los individuos y la influencia solo indirecta del gobierno" (FIEL, 1993, p. 7). Para ello, entonces se recomendaba arancelar y desarrollar un sistema de préstamos y becas que debe eliminarse en un lapso no mayor de cinco años a partir de la puesta en marcha de la reforma-. Como destaca Escalante Gonzalbo (2016), una de las tareas del Estado neoliberal consiste en crear mercados en que la dinámica consiste en que el propio Estado pague por los servicios, ya sea a través de particulares, mediante tickets o vales, o que lo haga directamente a las empresas a través de subsidios.

Además, también se intentaría incentivar la competencia entre universidades públicas y privadas a través de los vouchers o "cheques estudiantiles", ya que de este modo se tratan "en un pie de igualdad a universidades públicas y privadas y con una política completamente neutral entre universidades existentes y a crearse" (FIEL, 1993, p. 9).

En relación con esto, presentaban una redefinición del rol de la intervención del Estado en la universidad:

La educación superior es un ámbito en que el poder de policía del Estado deberá ejercerse al efecto de preservar las reglas del juego competitivas. Esta función, que tendrá un componente autoorganizado de la misma comunidad universitaria, implicará una supervisión de procedimientos (mecanismos de ingreso, evaluación, concursos, etc.) propios del quehacer universitario bien conocidos en la experiencia argentina e internacional. (FIEL, 1993, p. 10).

En este sentido, el futuro de las diferentes casas de estudio estaría supeditado a las decisiones del mercado y de los diferentes factores de poder que forman parte de la estructura social argentina. FIEL (1993) no descartaba entonces que

Algunas universidades públicas deberán sufrir profundas reestructuraciones de escala y orientación. El perfil del sistema universitario posterior a esta reforma es difícil de predecir, y dependerá entre otros factores clave del comportamiento de la demanda de educación superior y de la oferta laboral docente. Es posible que algunas universidades estatales mantengan un importante predicamento en el nuevo esquema, como resultado de las preferencias de los alumnos y docentes (...). La descentralización produciría 
ciertamente cambios importantes en el nivel de la oferta educativa, de la productividad, de la capacidad de elección del alumno con el aumento de responsabilidad involucrado, así como cierre y apertura de nuevas universidades, relocalizaciones, particiones de universidades que han superado la escala óptima, juntamente con una redistribución del ingreso más efectiva, el autofinanciamiento del sistema, la introducción de incentivos a la optimización del esfuerzo estudiantil, una formación de capital humano más acorde con las prioridades individuales y del mercado. (p. 12).

En resumen, ya desde comienzos de la década de 1990, FIEL considera que el sistema universitario argentino comprometía la competitividad de largo plazo de la economía argentina, un discurso similar al utilizado para justificar la privatización de las empresas públicas estatales (FIEL, 1993, p. 6).

Casi una década luego, basándose en los datos suministrados por el Programa de Mejoramiento del Sistema de Información Universitaria (PMSIU) de la Secretaría de Educación Superior del Ministerio de Educación de la Nación y del Anuario de Estadísticas Universitarias de 1999 y 2000, FIEL llevó adelante dos nuevas publicaciones, que retoman las propuestas de reforma esbozadas anteriormente.

En el documento Una educación para el Siglo XXI. Propuesta de reforma (2000) FIEL realizó un amplio diagnóstico y una serie de recomendaciones acerca de la educación en general, y el sistema universitario en particular sobre la base de una serie de datos publicados por diferentes dependencias del Estado. En particular, se nutrieron de los resultados de las evaluaciones educativas llevadas adelante por el Ministerio de Educación y Cultura (1999) en base las directrices del Educativos Data BASE de OCDE. Por otro lado, también recogieron información de la Dirección General de la Red Federal de Información docente (1998), del Censo Nacional de Docentes y Establecimientos educativos (1994) y de los resultados de la serie estadística de la Secretaría de Programación y Evaluación Educativa del Ministerio de Educación y Cultura (1997). De igual forma, a la hora de relacionar las políticas en educación superior con el gasto público, se recogieron datos de la Dirección Nacional del Gasto Social del Ministerio de Economía y Obras y Servicios Públicos de la Nación. 
Haciendo uso de un bagaje conceptual propio de la ortodoxia económica, los expertos de la fundación centraron sus críticas en torno a la "ineficiencia" del gasto estatal en educación superior y la "inequidad" que ello supone. Por un lado, se criticaba el alto costo de los graduados y la ineficiencia en la relación alumnos/graduados consecuencia "del grado de tolerancia a la mediocridad en la educación superior" (FIEL, 2000, p. 56) y en efecto, se argumentaba que no solo la cantidad de graduados era muy baja en relación con los ingresantes, sino que además se desconocía la calidad de esos graduados si se tienen en cuenta las deficiencias del sistema. Como hemos analizado anteriormente, la cuestión de la calidad fue uno de los puntos sobresalientes del programa de reformas de educación superior que financió el BM durante la década.

Así mismo, también destacaba la ineficiencia del sistema, consecuencia de la apertura de nuevas universidades públicas que durante los años 1990 se realizó por "criterios exclusivamente políticos" y cuyo financiamiento institucional sin condicionamientos no proveyeron incentivos para una mejora de la eficiencia educacional ${ }^{10}$. Por ello, sostenían que no existían incentivos para la libre competencia entre instituciones que condujera a una administración más eficiente del gasto, la mejora de la calidad y la adecuación de los programas a las demandas del mercado y el alumnado (FIEL, 2000, p. 58). Desde la Fundación se recomendaba que la asignación de recursos se centrara en los dispositivos que ofrece el propio mercado, que actuarían de forma "objetiva" a partir de la oferta y la demanda y el interés privado. $Y$ en este sentido, se desentendían de otras formas de asignación social de recursos, conscientes y colectivas, fruto de la discusión pública y democrática, orientada por la justicia y la integración social (Morduchowicz, 2004).

Un tercer elemento discutido desde las páginas de FIEL (2000) es el acceso irrestricto, al contrario de lo que se entiende por el término, era descripto como un ampliador de la inequidad social:

10 Entre 1989 y 2001 fueron creadas nueve universidades nacionales en todo el país: Universidad Nacional de La Matanza (1989), Universidad Nacional de Quilmes (1989), Universidad Nacional de General San Martín (1992), Universidad Nacional de General Sarmiento (1993), Universidad Nacional de La Rioja (1993), Universidad Nacional de la Patagonia Austral (1994), Universidad Nacional de Lanús (1995), Universidad Nacional de Tres de Febrero (1995) y Universidad Nacional de Villa María (1995). 
La provisión gratuita de educación superior para una gran parte del alumnado que está en condiciones de pagar sus estudios o de contraer préstamos, es al mismo tiempo una injusticia social y un incentivo indebido a la concurrencia de alumnos no preparados o insuficientemente motivados. (FIEL, 2000, p. 59).

Como destaca (Morduchowicz, 2004) las diversas propuestas de reforma de la educación universitaria de los expertos de FIEL reflejaban la lógica del discurso neoliberal, a partir de la aplicación a la educación de las reglas de juego del mercado donde las instituciones se convertían en corporaciones, los docentes en productores, los y las estudiantes en consumidores y el sistema educativo en un mercado nacional o global. En efecto, los principios de la gestión del Estado debían ser reemplazados por un nuevo modelo basado en el mercado, en donde la pertinencia entre la asimilación de organismos públicos y privados no es puesta en cuestión, como tampoco se pone en duda la eficacia de estos métodos en el sector privado (Dreyfus, 2012).

\section{Conclusiones: repliegue del Estado, radicalidad neoliberal y resistencia}

Con el fin de las ideologías que propició la caída de los socialismos reales y la hegemonía capitalista de finales de 1980, la década siguiente se caracterizó por ser un punto de inflexión político, cultural, económico y social que se expandió de la mano del neoliberalismo. En este marco, la disputa por la producción de sentidos fue ganada por los "expertos" en economía cuya demanda de conocimientos producidos en el ámbito privado se volvió constante, sobre todo a partir de la tecnificación en la toma de decisiones en las instituciones públicas (Brunner y Sunkel, 1995). Como destaca Dreyfus (2012), frente a las dificultades económicas, al aumento de la desocupación, la reducción de recursos públicos y la tendencia a la mundialización, la gestión del Estado se transformó en la administración de una empresa privada, lo que constituiría una receta milagrosa que conduciría a la resolución de los problemas que enfrentaban los Estados.

En un mundo donde la globalización y las redes del mercado como factores externos- y el poder económico marcaban limitaciones a la intervención del Estado, las recomendaciones de Fundación FIEL en el desarrollo de políticas en educación superior se encuentran 
en línea con el embate que sufrió la educación pública durante toda la década (Unzué y Emiliozzi, 2013).

El arancelamiento y los procesos de evaluación de las actividades desarrolladas por las universidades, en este sentido, fueron dos antídotos - recetados por los organismos internacionales y traducidos a nivel local por fundaciones privadas como FIEL - para reducir la ineficiencia y la inequidad del sistema universitario argentino y cuyos efectos se verifican en la LES (1995) y la creación de la CONEAU (1996) como ha sido descripto anteriormente.

Frente al repliegue del Estado, las recomendaciones de FIEL en política universitaria intentaron replicar el modelo económico en el sistema educativo, dejando de lado cualquier consideración acerca de la imbricación histórica entre las universidades argentinas y la sociedad, la construcción de ciudadanía y el desarrollo del país (Filmus, 1996). Por el contrario, las universidades públicas argentinas, por tradición gratuitas y de acceso irrestricto, debían convertirse en un bien que el Estado tenía que colocar al servicio de los flujos del mercado para la formación de recursos que los nutrieran.

Sin embargo, la radicalidad de las recomendaciones de FIEL —en gran medida- quedaron parcialmente en ello, puede arriesgarse, por el alto piso de movilización y organización del movimiento estudiantil y docente universitario argentino, que de forma continua a lo largo de la década de 1990 fue uno de los actores principales en oposición al modelo neoliberal (Argüello, 2006). Y fue gracias a esto que durante estos años las estructuras del sistema de educación superior no sufrieron grandes cambios, a diferencia de otros países de nuestro continente, como Chile o Brasil, donde efectivamente el sistema se ordenó a partir de la recreación de un mercado educativo donde, por un lado, el sistema público se convirtió en un espacio de circulación para las élites y el sector privado avanzó en la implantación y diversificación de la oferta educativa a lo largo de los países.

Entre las recomendaciones que efectivamente se tradujeron en decretos y leyes, podemos destacar el arancelamiento de los posgrados y la imposición de modelos de evaluación institucional, cuyo origen se puede rastrear a la llegada de financiamiento externo proveniente del BM y BID (Gordon, 2018). Como han considerado Unzué y 
Emiliozzi (2013) las universidades argentinas, en su diversidad como actores, con sus tradiciones y uso de la autonomía, son sujetos activos que actúan negociando, rechazando o alterando los intentos de aplicación de políticas para el sector en una compleja relación con el Estado. En síntesis, fue la trama de historias, tradiciones, equipos, investigadores, docentes y estudiantes la que permitió sostener las universidades nacionales frente a los embates del neoliberalismo. En Argentina, el sistema de educación superior y la universidad pública es uno de los nodos del articulado entre Estado y sociedad civil, fundamental para la construcción de la ciudadanía y el desarrollo económico.

\section{Referencias}

Acosta, Y., Giordano, V., y Soler, L. (2016). América Latina: nuestra. Cuadernos del pensamiento crítico latinoamericano, (36), Segunda época. http://biblioteca.clacso.edu.ar/clacso/ se/20160805024237/Cuaderno-PCL-N36.pdf

Albornoz, M. y Gordon, A. (2011). La política de ciencia y tecnología en Argentina desde la recuperación de la democracia (19832009). En M. Albornoz y J. Sebastián (Eds.), Trayectorias de las políticas científicas y universitarias de Argentina y España (pp. 1-46). Consejo Superior de Investigaciones Científicas, CSIC.

Ansaldi, W. (2017). Arregladitas como para ir de boda. Nuevo ropaje para las viejas derechas. Theomai, (35), 22-51. http://revistatheomai.unq.edu.ar/numero_35/2.\%20Ansaldi.pdf

Ansaldi, W. y Giordano, V. (2012). América Latina, la construcción del orden: de las sociedades de masas a las sociedades en proceso de reestructuración. Ariel.

Ansaldi, W. y Soler, L. (2015). Derechas en América Latina en el siglo XXI. En R. Carbone y L. Soler (Eds.), Des(Cartes). Estampas de la derecha paraguaya (pp. 15-27). Punto de Encuentro.

Argüello, S. B. (2006). Movimiento estudiantil, política de educación superior y exclusión educativa. Cuadernos de la Facultad de Humanidades y Ciencias Sociales, (30), 13-40. http://www. scielo.org.ar/pdf/cfhycs/n30/n30a01.pdf

Banco Mundial. (1995). La educación superior en América Latina. Cuestiones sobre eficiencia y equidad. Banco Mundial. 
Beltrán, G. J. (2011). Escenas de la vida corporativa. Conocimiento y toma de decisión en las organizaciones. Apuntes CECYP, (19), 103-130. https://publicaciones.sociales.uba.ar/index. $\mathrm{php} /$ apuntescecyp/article/view/4096

Bobbio, N., Matteucci, N., y Pasquino, G. (1994). Diccionario de Política, L-Z. Siglo XXI Editores.

Bonnell, V. E. (1980). The uses of theory, concepts, and comparison in historical sociology. Comparative Studies in Society and History, 22(2), 156-173. https://doi.org/10.1017/ S0010417500009270

Bonnet, A. (2008). La hegemonía menemista. Prometeo.

Botto, M. (2011). Think tanks en América Latina: Radiografía comparada de un nuevo actor político (Documentos de trabajo 60). Facultad Latinoamericana de Ciencias Sociales, FLACSO.

Braudel, F. y Mendoza, J. G. (1970). La historia y las ciencias sociales, Vol. 22. Alianza editorial.

Brunner, J. y Sunkel, G. (1995). Conocimiento, sociedad y política. Facultad Latinoamericana de Ciencias Sociales, FLACSO.

Camou, A. (1997). Los consejeros del príncipe. Saber técnico y política en los procesos de reforma económica en América Latina. Nueva Sociedad, (152), 54-67. https://static.nuso.org/media/articles/downloads/2639_1.pdf

Carrera, G. (1976). La renovación de los estudios históricos: El caso de Venezuela. Ediciones Sep/Setenta.

Castiglioni, F. (1996). Argentina. Política y economía en el menemismo. Nueva Sociedad, (143), 6-14. https://static.nuso.org/media/ articles/downloads/2496_1.pdf

Ciencia: Hora de cuentas. (1996, julio 11). La Nación.

Del Bello, J. C. (1996a). Informe de gestión 1. Ministerio de Cultura y Educación, Secretaría de Ciencia y Tecnología.

Del Bello, J. C. (1996b). Informe de Gestión 2. Ministerio de Cultura y Educación, Secretaría de Ciencia y Tecnología.

Dreyfus, F. (2012). La invención de la burocracia. Servir al Estado en Francia, Gran Bretaña y Estados Unidos (Siglos XVIII-XX). Biblos.

Escalante, F. (2016). Historia mínima del neoliberalismo. El Colegio de México, Turner. 
Filmus, D. (1996). Estado, sociedad y educación en la Argentina de fin de siglo: proceso y desafíos. Troquel.

Filmus, D. (2016). Una década de transformaciones en América Latina. Consejo Latinoamericano de Ciencias Sociales, CLACSO.

Fischer, K. y Plehwe, D. (2013). Redes de think tanks e intelectuales de derecha en América Latina. Nueva Sociedad, (245), 70-86. https://static.nuso.org/media/articles/downloads/3941_1.pdf

Fundación de Investigaciones Económicas Latinoamericanas, FIEL (s.f). Acerca de. http://www.fiel.org/acercade

Fundación de Investigaciones Económicas Latinoamericanas. (1993). Documento de Trabajo $N^{\circ} 38$. La descentralización de la educación superior: elementos de un programa de reforma. Buenos Aires.

Fundación de Investigaciones Económicas Latinoamericanas. (2000). Una educación para el Siglo XXI. Buenos Aires.

Fundación de Investigaciones Económicas Latinoamericanas. (2003). Documento de Trabajo $N^{\circ} 79$. Educación universitaria. Aportes para el debate acerca de su efectividad y equidad. Buenos Aires.

Giménez, M. J. (2021). Navegar el Atlántico a contramarea: La Fundación Internacional para la Libertad y la agenda liberal en la ofensiva en América Latina (2002-2016) [Tesis Doctoral]. Universidad Estadual de Campinas.

Gordon, A. (2018). Estudio de las políticas de ciencia, tecnología y educación superior en Argentina, Brasil y Chile desde mediados de la década de 1990 [Tesis doctoral no publicada]. Universidad de Buenos Aires.

Giordano, V. (2014). ¿Qué hay de nuevo en las "nuevas derechas"? Nueva sociedad, (254), 46-56. https://static.nuso.org/media/articles/downloads/4068_1.pdf

Grassetti, J. y Prego, F. (2017). Think tanks, intelectuales y derechas. El rol de la Fundación para el Análisis y los Estudios Sociales (FAES) en Venezuela y Argentina (2015-2017). MILLCAYAC, Revista Digital de Ciencias Sociales, 4(7), 121-140. https://revistas. uncu.edu.ar/ojs/index.php/millca-digital/article/view/1016

Heredia, M. (2011). La hechura de la política económica. Los economistas, la convertibilidad y el modelo neoliberal. En A. Puc- 
ciarelli (Ed.), Los años de Menem: la construcción del orden neoliberal (pp. 179-220). Siglo XXI Editores.

Lorenc Valcarce, F. (2002). Agenda política, producción de sentido y conflictos sociales en la Argentina. El último año del gobierno de Menem. En B. Levy (Ed.), Crisis y conflicto en el capitalismo latinoamericano: lecturas políticas (pp. 29-56). Consejo Latinoamericano de Ciencias Sociales, CLACSO.

McGann, J. G. y Whelan, L. C. (2020). Global think tanks: Policy networks and governance. Routledge.

Mercado, A. B. (2017). Think tanks, democracia y partidos políticos. El Instituto de Ciencia Política Hernán Echavarría Olózaga durante la reforma constitucional colombiana (1986-1992). MILLCAYAC, Revista Digital de Ciencias Sociales, 4(7), 49-70. https://revistas.uncu.edu.ar/ojs/index.php/millca-digital/ article/view/1017

Mitchell, P. (2021). Think tanks y la diplomacia académica de la Fundación Ford en Argentina (1975-1983). Estudios Sociales Contemporáneos, 1(24), 48-72. https://doi.org/10.48162/ rev.48.002

Morales, J. J. (2021). [Reseña] Global Think Tanks. Policy Networks and Governance, de James G. McGann y Laura C. Wheelan. Estudios Públicos, (162), 187-194. https://www.cepchile.cl/cep/site/docs/20210531/20210531110631/rev162_ jjmorales.pdf

Morduchowicz, A. (2004). Discusiones de economía de la educación. Losada.

Morresi, S. (28-30 de julio de 2010). Los expertos en economía y las ideas neoliberales sobre la democracia latinoamericana. V Congreso Latinoamericano de Ciencia Política. Asociación Latinoamericana de Ciencia Política, Ciudad Autónoma de Buenos Aires, Argentina.

Oszlak, O. y Gantman, E. (1994). Los A.G.: la creación de un cuerpo gerencial de elite en el sector público argentino. https:// n9.cl/7afw5

Portantiero, J. C. (1988). La producción de un orden: Ensayos sobre la democracia entre el Estado y la sociedad. Nueva Visión.

Pucciarelli, A. (2011). Los años de Menem: la construcción del orden neoliberal. Siglo XXI Editores. 
Ramírez, H. (2007a). Hasta que los intereses nos separen: conflictos en FIEL y la Fundación Mediterránea. H-industri@, Revista de Historia de la Industria Argentina y Latinoamericana, 1(1). https://dialnet.unirioja.es/descarga/articulo/5009821.pdf

Ramírez, H. (2007b). Corporaciones en el poder. Institutos económicos y acción política en Brasil y Argentina: IPÊS, FIEL y Fundación Mediterránea. Lenguaje claro Editora.

Scargiali, E. A. (2018). Fieles al mercado: breve análisis de las trayectorias de los expertos de Fundación FIEL durante la década neoliberal en Argentina (1989-2001). Revista Internacionales. Revista en Ciencias Sociales del Pacifico Mexicano, 4(8), 54-76. http:// cainternacionales.com/revista/articulos/vol-4/num-8/pdf/ RI8\%20Scargiali\%20E\%20-\%20Fieles\%20al\%20mercado.pdf

Soler, L., Giordano, V., y Saferstein, E. (2018). Las derechas y sus raros peinados nuevos. Apuntes de Investigación del CECYP, (30), 171-191. https://apuntescecyp.com.ar/index.php/apuntes/ article/view/689/527

Svampa, M. (2008). Notas provisorias sobre la sociología, el saber académico y el compromiso intelectual. En M. Svampa y V. Hernández (Eds.), Gérard Althabe: Entre dos mundos. Reflexividad y compromiso. Prometeo. http://maristellasvampa.net/ archivos/ensayo41.pdf

Tenti Fanfani, E. (2004). Prólogo. En A. Morduchowicz (Ed.), Discusiones de economía de la educación (pp. 11-15). Losada.

Thompson, A. (1994). Think tanks en la Argentina. Conocimiento, instituciones y política. Centro de Estudio de Estado y Sociedad, CEDES.

Unzué, M. (2013). Autonomía, evaluación y políticas públicas. Tendencias y límites en los sistemas universitarios de Argentina y Brasil. En M. Unzué y S. Emiliozzi (Eds.), Universidad y políticas públicas ${ }_{i}$ En busca del tiempo perdido? Argentina y Brasil en perspectiva comparada (pp. 9-49). Imago mundi.

Unzué, M. y Emiliozzi, S. (2013). Prólogo. En M. Unzué, y S. Emiliozzi (Eds.), Universidad y políticas públicas iEn busca del tiempo perdido? Argentina y Brasil en perspectiva comparada (pp. 1-9). Imago Mundi.

Uña, G., Cogliandro, G., y Labaqui, J. (2004). Políticas públicas y toma de decisiones: los think tanks en Argentina. Fundación Konrad Adenauer. 
Uña, G. y Garcé, A. (Eds.), (2006). Think tanks y políticas públicas en Latinoamérica: dinámicas globales y realidades regionales. Prometeo Libros.

Vommaro, G. (2014). Meterse en política: la construcción de PRO y la renovación de la centroderecha argentina. Nueva Sociedad, (254), 57-72. https://ri.conicet.gov.ar/handle/11336/44980 